the jury had not been sitting on this case for over 30 years (Woodruff et al, 1967) before coming to a verdict. Leff's early work (1978) identifies the heart of the problem: joining anxiety and depression upsets the epidemiologists, pharmacologists and researchers who would like anxiety and depression to stay apart, but for patients and clinicians these emotions are often joined together intimately. Let them at least have a trial marriage before condemning them indefinitely to live in nosological sin.

Leff, J. P. (1978) Psychiatrists' versus patients' concepts of unpleasant emotions. British Journal of Psychiatry, 133, 306-313.

Seivewright, H., Tyrer, P. \& Johnson, T. (1998)

Prediction of outcome in neurotic disorder: a 5-year prospective study. Psychological Medicine, 28, II49-1157.

Tyrer, P. (200I) The case for cothymia: mixed anxiety and depression as a single diagnosis. British journal of Psychiatry, 179, 191-193.

_, Seivewright, H., Simmonds, S. et al (200I) Prospective studies of cothymia (mixed anxietydepression): how do they inform clinical practice? European Archives of Psychiatry and Neuroscience, in press.

Woodruff, R., Murphy, G. \& Herjanic, M. (1967) The natural history of affective disorders: I. Symptoms of 72 patients at the time of index hospital admission. Journal of Psychiatric Research, 5, 255-263.

P.Tyrer Department of Public Mental Health, Division of Neuroscience and Psychological Medicine, Imperial College School of Medicine, St Mary's Campus, Paterson Centre, 20 South Wharf Road, London W2 IPD, UK

\section{Cross-cultural training in psychiatry}

Farooq (2001) has highlighted some inadequacies in the psychiatric training imparted in developing countries. He has suggested the need for a total paradigm shift in training to address these problems. As a psychiatric trainee having initially trained in a developing country and now undergoing training in the UK, I cannot agree more with this suggestion. However, I wish to make some further points.

Some doubts are expressed about the suitability of training in developed countries for psychiatrists who will ultimately work in developing countries. There may be some disparities between training needs and the training obtained, but this is only to be expected, given the nature and extent of the differences in psychiatric practice. But cross-cultural training exchange helps develop the insight into both worlds that is required to be able, one hopes, to make some contribution towards changing psychiatric practice in the developing world.

Conversely, Britain is a multicultural society with many ethnic groups, some members of which formerly lived in the developing world, which in turn might influence their current customs and practices. It might be a good idea for British psychiatric trainees (and indeed trainees from other developed countries) to obtain some training in the developing world. This would give greater insight into the cultural variations in psychiatry. I am sure many would find this an exciting and rewarding prospect. After all, one of the best ways of learning about other cultures is to be among them and to partake of the local customs and traditions.

Farooq, S. (200I) Psychiatric training in developing countries (letter). British Journal of Psychiatry, 179, 464.

H. Subramaniam Gardner Unit, Mental Health Services of Salford, Prestwich Hospital, Bury New Road, Prestwich, Manchester M25 3BL, UK

\section{Substance misuse among people with schizophrenia: similarities and differences between the UK and France}

We read with interest the paper by Duke $e t$ al (2001) reporting on non-alcohol substance misuse among people with schizophrenia. We recently conducted a study addressing that question among patients with schizophrenia from the Parisian suburbs, using the Composite International Diagnostic Interview for DSM-III-R diagnoses of substance abuse or dependence (Dervaux et al, 2001). We found that
$41 \%$ of the patients had a lifetime history of substance abuse or dependence, including $30 \%$ on a drug other than alcohol $(\nu$. $16 \%$ in the Duke et al study). As in Duke et al's study, in our study the patients with substance abuse were younger than nonabusers. We also found no difference in respect of psychiatric symptoms using the Positive and Negative Syndrome Scale, mean age of first psychiatric treatment or mean number of previous hospitalisations. The patterns of consumption were slightly different for cannabis $(27 \%$ of the patients in Paris $v .18 .7 \%$ in London) and opiates $(7 \% \quad v .5 .3 \%$, respectively). The main difference concerned the misuse of cocaine and crack, less common in France $(1 \%)$ than in the UK $(8.7 \%)$, and considerably less than in North American studies (ranging from 20 to $30 \%$; Cantor-Graae et al, 2001). Although methodological issues could explain some of these differences (e.g. the use of different assessment instruments; mean age of the subjects was 34.7 years in our study $v .50 .3$ years in the English study), these patterns most probably reflect the different availability of drugs in these countries. Taken together, these observations do not argue in favour of the self-medication hypothesis but instead emphasise the role of alternative, and probably multiple, factors leading to substance misuse in schizophrenia.

Cantor-Graae, E., Nordström, L. G. \& McNeil,T. E. (200I) Substance abuse in schizophrenia: a review of the literature and a study of correlates in Sweden. Schizophrenia Research, 48, 69-82.

Dervaux, A., Bayle, F.-J., Laqueille, X., et al (200I) is substance abuse in schizophrenia related to impulsivity, sensation seeking or anhedonia? American Journal of Psychiatry, 158, 492-494.

Duke, P. J., Pantelis C., McPhillips, M. A., et al (200I) Comorbid non-alcohol substance misuse among people with schizophrenia. Epidemiological study in central London. British Journal of Psychiatry, I79, 509-513.

A. Dervaux, F. J. Bayle, M.-O. Krebs Service Hospitalo-Universitaire de Santé Mentale et Thérapeutique, Prs H. Lôo et J.-P. Olié, Centre Hospitalier Sainte-Anne, I Rue Cabanis, F-75014, Paris, France 